ÉGYPTE

monde arabe

\section{Égypte/Monde arabe}

$34 \mid 1998$

Droits d'Égypte : histoire et sociologie

\title{
Réception du droit français sous les britanniques en Égypte : un paradoxe?
}

Jan Goldberg

Traducteur : Nathalie Bernard-Maugiron

\section{(2)enEdition}

Journals

Édition électronique

URL : https://journals.openedition.org/ema/1493

DOI : 10.4000/ema.1493

ISSN : 2090-7273

Éditeur

CEDEJ - Centre d'études et de documentation économiques juridiques et sociales

Édition imprimée

Date de publication : 31 décembre 1998

Pagination : $67-80$

ISSN : 1110-5097

Référence électronique

Jan Goldberg, « Réception du droit français sous les britanniques en Égypte : un paradoxe ? », Égypte/ Monde arabe [En ligne], 34 | 1998, mis en ligne le 08 juillet 2008, consulté le 07 juillet 2022. URL : http:// journals.openedition.org/ema/1493 ; DOI : https://doi.org/10.4000/ema.1493

Ce document a été généré automatiquement le 7 juillet 2022.

Tous droits réservés 


\title{
Réception du droit français sous les britanniques en Égypte : un paradoxe?
}

\author{
Jan Goldberg
}

Traduction : Nathalie Bernard-Maugiron

\section{NOTE DE L'ÉDITEUR}

Traduit de l'anglais

1 Les comparatistes recourent à divers types de catégories pour classer les systèmes juridiques en ce qu'ils appellent des familles de droit. David (1964, p. 15-17), par exemple, était en faveur d'une classification en fonction de l'arrière-fond idéologique du système juridique. Arminjon, Nolde et Wolff (1950-1952, vol. 1, p. 47-49) furent les premiers à proposer que les systèmes juridiques soient étudiés, comparés et classifies sur la base de leur seule substance et non en fonction de facteurs externes comme la géographie, le climat, la mentalité ou l'idéologie. Ils ont ainsi répertorié sept familles de systèmes juridiques : française, germanique, Scandinave, anglaise, russe, islamique et hindoue, auxquelles Zweigert et Kötz (1987, p. 75) ajoutèrent « les systèmes de l'Extrême-Orient ».

2 La famille de droit français comprend de nos jours une variété d'États, pays ou régions à travers le monde. Les systèmes juridiques de ces entités, leurs tribunaux et leurs Codes suivent à des degrés divers les Codes napoléoniens de la France postrévolutionnaire ou les ont suivis dans le passé, se développant ensuite de façon autonome sans toutefois remettre totalement en question leurs fondements juridiques. Pour Rheinstein (1987, p. 78), les membres actuels de la famille de droit français sont la Belgique, le Luxembourg, Monaco, Haïti, l'Italie, l'Espagne, le Portugal, les Pays-Bas, les pays d'Amérique latine, le Québec, les anciennes colonies françaises et belges en Afrique, l'Indonésie, les pays du Maghreb (Maroc, Tunisie, Algérie), ainsi que l'Égypte, 
le Liban et la Syrie. Zweigert et Kötz (1987, p.119-121) y incluent également la Louisiane.

3 Une famille de droit peut voir le nombre de ses membres augmenter ou au contraire diminuer. Le procédé par lequel une entité politique devient membre d'une famille de droit est appelé réception juridique, ou simplement réception. Selon Hirsch (1981, p.123-124), le trait le plus caractéristique d'une réception est l'adoption totale ou partielle d'un droit, de lois, de Codes ou de structures judiciaires étrangers. Cela entraîne généralement la traduction de ces textes dans la langue du pays récepteur, puis leur promulgation en tant que lois propres à cet État.

4 C'est dans les années 1870 que l'Égypte fit son entrée dans la famille du droit français, par un processus de réception des Codes français. En 1876, tout d'abord, les tribunaux mixtes (mahâkim mukhtalita), qui venaient juste d'être créés, furent dotés de 6 Codes Code civil, Code de commerce, Code de commerce maritime, Code de procédure civile et commerciale, Code pénal et Code d'instruction criminelle ${ }^{1}$ - qui reprenaient de façon quasi intégrale des pans entiers des Codes français correspondants.

5 Maunoury, le juriste français d'Alexandrie à qui l'on doit l'élaboration de ces Codes mixtes, se serait inspiré non seulement du droit français, mais également des droits italien, belge et islamique (Khânkî, 1939, p. 88). Comme, à l'époque, les droits italien et belge étaient eux-mêmes très proches du droit français, il est difficile de connaître avec certitude l'origine exacte d'une règle. Les quelques principes tirés de la sharî'a qui figurent dans les Codes mixtes concernent des domaines inconnus du droit français, comme le waqf (bien de mainmorte ou fondation pieuse).

6 La compétence pénale des tribunaux mixtes a été boycottée dans une très large mesure par les puissances consulaires (Brinton, 1968, p.115). Leurs attributions dans les domaines civil et commercial couvraient tous types de litiges, y compris ceux relatifs à la terre, survenus entre des étrangers et des nationaux, ou entre étrangers de nationalité différente. Tout ce qui concernait le statut personnel, la famille ou les successions (Brinton, 1968, p. 60) restait toutefois du ressort des tribunaux shar'î (mahâkim shar'iyya), des tribunaux des communautés non musulmanes d'Égypte ou des tribunaux consulaires, selon les cas (Brinton, 1968, p. 159-163).

7 En 1883, un an après l'arrivée des Britanniques en Égypte, des tribunaux nationaux (mahâkim ahliyya) furent mis en place et chargés de régler tous les litiges civils, commerciaux ou pénaux survenus entre Égyptiens. Ces tribunaux furent dotés à leur tour de leurs propres Codes, inspirés des Codes mixtes. Fruits des efforts conjoints d'une équipe de juristes français, italiens et égyptiens - dont la majorité occupait des fonctions au sein des tribunaux mixtes ou du gouvernement égyptien (Khânkî, 1939, p. 92) ${ }^{2}$-, ces Codes nationaux furent d'abord rédigés en français puis ensuite seulement traduits en arabe.

8 Par l'introduction de ces Codes mixtes et nationaux, le droit français devenait donc source première de la législation. Ce fut le début d'un processus de réception juridique dont le couronnement fut le célèbre Code civil de 1948 de 'Abd al-Razzâq al-Sanhûrî. Ce Code, toujours en vigueur aujourd'hui, devait remplacer les Codes civils mixtes et nationaux. Comme le Code Sanhûrî eut à son tour une grande influence à travers le Moyen-Orient, Krüger (1987) estime qu'il a créé une véritable famille de droit égyptien, à l'intérieur de la famille française de droit ${ }^{3}$. 
9 Le début du processus de réception, en 1876, marqua l'aboutissement d'un long cheminement vers l'adhésion à la famille de droit français, commencé avant même la promulgation des Codes napoléoniens en France, dès l'époque de l'occupation française. Sans toucher au système juridique islamique en tant que tel, les Français avaient ainsi toutefois déjà introduit cinq innovations substantielles en droit commercial : des tribunaux commerciaux spéciaux; le concept de juridictions mixtes ; le recrutement de commerçants de façon paritaire au sein des communautés musulmanes et non musulmanes pour siéger dans ces tribunaux; la preuve par écrit dans les transactions civiles et commerciales et la nomination de dhimmî à des postes clés comme celui de président de tribunal de commerce (Laurens et al., 1989, p. 117).

Bien que limitées au droit commercial, ces innovations eurent cependant un impact bien au-delà de ce seul domaine. Ainsi, le fait de nommer des dhimmî à de hautes charges dans les domaines juridictionnel, administratif et du maintien de l'ordre, entraîna une quasi-suspension de la conception islamique de la hiérarchie sociale. Pour la première fois, des litiges entre musulmans et dhimmîn n'étaient pas automatiquement tranchés par un juge de confession musulmane. Ce fut le point de départ d'un processus de transformation des élites du droit, ou, pour reprendre les termes de Weber, des « honoratiores dudroit».

11 Pour Weber, les systèmes juridiques diffèrent davantage par leur infrastructure judiciaire que par leurs normes, et la nature de l'infrastructure judiciaire d'un système juridique donné est déterminée par ses honoratiores (Rheinstein, 1954, p. 198-223). Par conséquent, tout changement au niveau de ces honoratiores - et a fortiori leur remplacement - se répercute au niveau du système juridique.

12 Ce processus de transformation des élites du droit avait donc précédé la mise en place des tribunaux mixtes et nationaux. L'occupation française aurait pu n'indiquer que la route à suivre (Goldberg, 1997). En choisissant d'envoyer des missions d'étudiants en France, dès les années 1820, pour étudier le système juridique français (Botiveau, 1989, p. 234), l'Égypte se tourna toutefois résolument vers le droit français. Deux facteurs avaient convergé : une communauté importante et influente de technocrates français et un nouveau dirigeant très réceptif à l'expertise française.

$13 \mathrm{Au}$ début des années 1830, l'Égypte adopta de façon indirecte les lois militaires françaises en important les Codes militaires ottomans, eux-mêmes inspirés du droit français (Fahmy, 1997, p.137-141). Le siyâsatnâme de1837, loi qui devait servir de fondement à toute l'administration de l'État jusqu'à la fin du XIXe siècle, avait été élaboré en suivant les conseils d'un expert français, chargé par Muhammad 'Alî d'étudier l'appareil gouvernemental (Hamed, 1995, p. 79)4. Quant au qânûn almuntakhabât de1844, compilation de lois pénales, il reprenait diverses dispositions du Code pénal français de 1810 (Peters, 1997, p. 73-74)

14 Le concept de tribunal commercial survécut à l'occupation française. Des tribunaux mixtes commerciaux fonctionnèrent ainsi dans les années 1820 et 1830 (Reimer, 1997, p. 81-82) avant d'être réorganisés au milieu des années 1840 et d'être appelés majâlis altujjâr ${ }^{6}$. Établis au Caire et à Alexandrie, ils ressemblaient aux tribunaux commerciaux de l'époque de l'occupation française, étant, comme eux, composés de marchands et présidés par un fonctionnaire d'État. Ils en différaient toutefois notamment par le fait que, pour la première fois, ils étaient mixtes par la nationalité et non par la religion. Chargés de tout litige entre commerçants, ils privèrent les tribunaux islamiques et consulaires de leur compétence juridique en matière commerciale. Ces majâlis furent les 
précurseurs des tribunaux mixtes aussi bien par leur structure que par leur composition. Ils servirent également de «cobayes" pour tester de nouveaux instruments comme la procédure d'appel ou l'application du droit civil et commercial français.

Si l'on garde à l'esprit tous ces développements, il semble donc moins paradoxal que le droit français ait été introduit sous les Britanniques. L'hypothèse de Brown (1997, p. 48) selon laquelle l'Égypte aurait adopté le droit français pour ne pas se voir imposer le droit anglais, peut expliquer que les tribunaux nationaux et leurs Codes suivirent le modèle des tribunaux mixtes et de leurs Codes. Lord Cromer, qui servit en Égypte de 1883 à 1907 comme agent britannique et Consul général de Grande-Bretagne, explique pourquoi il était trop tard pour que les Britanniques puissent mettre en place une alternative au droit français :

«Le droit et la procédure français avaient déjà pris racine en Égypte. Les Codes utilisés par les tribunaux mixtes étaient français. Tous les jeunes Égyptiens qui avaient reçu une formation juridique avaient suivi des études en France. Il était de ce fait inévitable que les nouveaux tribunaux s'inspirent d'un modèle français plutôt qu'anglais. » (Cramer, 1908, vol. 2, p. 516) ${ }^{7}$

Cromer regrette que les Britanniques n'aient pas été en mesure de mettre en œuvre une alternative, sous la forme d'un Code véritablement égyptien, adapté aux circonstances et aux besoins de l'Égypte. Pour lui, les tribunaux mixtes et leurs Codes étaient "trop européens pour le pays» (Cromer, 1908, vol. 2, p. 319) ou, en d'autres termes, ils n'étaient pas assez égyptiens. Cromer estime ainsi que le Code civil des tribunaux mixtes n'était qu'une copie du Code civil français, inadapté à la réalité de l'Égypte et, qui plus est, appliqué par des juges presque tous ignorants des us et coutumes égyptiens. Le peuple égyptien, de son côté, ignorait tout du droit qui lui était appliqué, situation qui risquait d'être gravement préjudicielle.

On peut se demander ce que Cromer entendait par «modèle anglais » comme alternative au modèle français d'organisation juridictionnelle. Par ailleurs, le Code civil mixte était-il vraiment étranger à l'Égypte?

Cromer, qui, avant d'être envoyé en Égypte, avait servi dans la haute administration en Inde (Mansfield, 1973, p. 59-61), se réfère à l'expérience britannique dans ce dernier pays et au Soudan (Cromer, 1908, vol.2, p. 319 et 516-517), où droit et procédure auraient été adaptés aux circonstances et aux besoins spécifiques des deux pays.

19 Les principes britanniques d'administration juridique avaient été introduits bien avant que la Couronne britannique ne remplace officiellement la Compagnie orientale des Indes comme gouverneur, en 1858 (Patra, 1962). Les Moghols ayant octroyé, en 1765, le droit de collecter des impôts et d'administrer les affaires de droit privé à la Compagnie orientale des Indes, Warren Hastings, gouverneur général du Bengal, pu mettre en place, en 1772, un système judiciaire pour ce qu'on appelait les mofussil, les territoires $\mathrm{du}$ Bengal, de Bihar et d'Orissa. Ces régions avaient une population socialement plus diversifiée que celle des villes-présidences de Bombay, Calcutta et Madras. Alors que le droit anglais était appliqué par des tribunaux anglais dans ces dernières, il était prévu pour les tribunaux de la Compagnie dans les mofussil que :

« Dans tout litige concernant une affaire d'héritage, de mariage, de caste et autres tradition ou institution religieuses, les lois du Coran pour les Mahométans et celles $\mathrm{du}$ Shaster pour les Gentoos devront toujours s'appliquer. $»^{8}$ 
À partir de 1781, les affaires de succession en général furent également de la compétence de la loi islamique ou hindoue. Dans les autres cas, le juge devait décider conformément à « la justice, l'équité et à la bonne conscience " ${ }^{9}$. Ce dernier principe fut également appliqué dans le Soudan anglo-égyptien (Mustafa, 1971), qui était de jure un condominium, mais se voyait de facto soumis à l'administration britannique. En 1899, un an à peine après avoir conquis le Soudan, les Britanniques introduisirent un Code pénal et un Code de procédure pénale, inspirés des lois indiennes équivalentes (Ismail, 1982, p. 1). Un an après, «l'ordonnance de justice civile 1900 » prévoyait dans son article 3 que :

« $\mathrm{Si}$, à l'occasion d'un litige ou à toute autre occasion, est soulevée devant une juridiction civile une affaire de succession, d'héritage, de testament, de legs, de don, de mariage, de divorce, de relations familiales ou de constitution de waqf, la règle applicable devra être: a) toute coutume applicable aux parties concernées, à condition qu'elle ne soit pas contraire à la justice, à l'équité et à la bonne conscience (good conscience) et qu'elle n'ait pas été amendée ou abolie par la présente ordonnance ou par toute autre loi, et n'ait pas été déclarée nulle par décision d'une autorité compétente; b) la loi mahométane, dans les cas où les parties sont mahométanes, sauf si cette loi a été modifiée par une des coutumes auxquelles il a été fait allusion précédemment. $»^{10}$

21 Pour toutes les autres affaires civiles, l'article 4 de la même ordonnance prévoyait que «le tribunal devra statuer en application des principes de justice, d'équité et de bonne conscience $»^{11}$.

Alors qu'en Inde un mouvement de codification entraîna dans la seconde moitié du $\mathrm{XIX}^{\mathrm{e}}$ siècle, probablement sous l'influence universelle des Codes napoléoniens, une plus grande réception du droit anglais et les débuts de la formation d'un droit «anglomahométan " (David, 1964, p. 506-509), au Soudan, c'est à partir des principes de " justice, équité et bonne conscience », tels qu'interprétés par les tribunaux soudanais, que le droit anglais parvint à se frayer un chemin. Pour Ismail, ce serait surtout vrai pour la période postérieure à 1919, les tribunaux ayant avant cela fondé leurs décisions sur la base d'une comparaison entre le droit égyptien et le droit anglais (Ismail, 1982, p. 15, 33, 44).

Comme ils ne pouvaient pas instaurer en Égypte un système judiciaire totalement nouveau, les Britanniques cherchèrent à modifier autant que possible le système en place. Cromer invoque trois raisons qui justifieraient qu'une introduction progressive de réformes mineures ait été préférée à un changement radical: «le caractère du peuple », «l'impossibilité de créer rapidement un pouvoir judiciaire compétent » et les problèmes spécifiques dus à " une période de transition d'un gouvernement arbitraire vers un État de droit (Reign of Law)» (Cromer, 1908, vol. 2, p. 517).

La stratégie britannique d'anglicisation à partir de l'intérieur porta plus particulièrement sur le droit pénal, procédure et instruction. Saroufim prétend que pendant toute la durée de leur occupation de l'Égypte, les Anglais ont essayé et ont effectivement réussi à rendre conforme le système pénal égyptien au leur. Dans ce domaine, la contribution britannique au système juridique égyptien aurait été aussi importante que celle des Français (Saroufim, 1949). Les travaux de Saroufim ne permettent toutefois pas de démontrer que les changements que les Britanniques réussirent à introduire auraient effectivement été au-delà de simples modifications d'ordre procédural, justifiées par des objectifs de simplification et de rationalisation de 
la procédure. C'est ainsi par exemple que les Britanniques supprimèrent le juge d'instruction, chargé des premières investigations judiciaires (Scott, 1899, p. 249).

«Pourquoi les Britanniques n'ont-ils pas fait en Égypte ce qu'ils ont fait en Inde?», Berger (1957, p. 26-27) s'est posé la question et a tenté d'y répondre. D'un côté, les Britanniques seraient restés en Inde beaucoup plus longtemps qu'en Égypte et, d'un autre côté, ils auraient eu les coudées beaucoup plus franches dans le premier cas, leur présence en Inde datant des débuts de l'impérialisme, alors que peu de temps après leur arrivée en Égypte ils se trouvaient déjà confrontés à l'anticolonialisme et au nationalisme égyptiens.

Selon Berger, l'intérêt de la Grande-Bretagne pour l'Égypte ne venait qu'en deuxième position par rapport à son intérêt pour l'Inde. L'Égypte n'était considérée que comme une tête de pont entre l'Angleterre et l'Inde, et le contrôle de l'Égypte n'était stratégiquement nécessaire que pour la sécurité des communications à l'intérieur de l'Empire. Pour parvenir à cette fin, la société égyptienne n'avait pas besoin d'être pénétrée aussi profondément que la société indienne. Les administrateurs britanniques estimaient ainsi qu'ils n'avaient pas vraiment besoin d'atteindre les régions provinciales mais qu'ils pouvaient se confiner aux administrations centrales dans la capitale et dans les grandes villes.

L'argument de Berger relatif à la durée comparative de la présence britannique en Égypte et en Inde est certainement exact. Son approche laisse toutefois à désirer en ce qui concerne la question des intérêts de la Grande-Bretagne, car elle sous-entend que c'est volontairement que les Britanniques n'ont pas fait en Égypte ce qu'ils ont fait en Inde. Cromer a donné l'impression inverse. Berger lui-même a fait remarquer que l'impérialisme en général était déjà sur son déclin et que, par conséquent, la position des Britanniques en Égypte n'avait pas pu être aussi forte qu'elle l'avait été en Inde. L'impérialisme britannique, dans sa version colonialiste, était confronté à une opposition en Égypte, mais également en Angleterre. De plus, le colonialisme britannique s'est trouvé en compétition permanente avec son grand rival français. Par conséquent, la question n'est pas de savoir pourquoi les Britanniques n'ont pas fait en Égypte ce qu'ils ont fait en Inde, mais pourquoi ils n'ont pas pu faire en Égypte ce qu'ils ont pu faire en Inde.

On ne peut pas répondre à cette question en se référant simplement au déclin de l'impérialisme et aux conséquences qui en ont été mentionnées. L'exemple du Soudan, où les Britanniques ont réussi à appliquer leurs expériences "indiennes", s'oppose à une telle explication sommaire. Le dernier facteur explicatif de Berger est plus convaincant. Si les Britanniques n'ont pas pu appliquer en Égypte leur politique angloindienne, c'est parce que : « [ils] ont occupé l'Égypte après un siècle environ d'influence française au niveau de l'administration publique, de l'éducation et des relations économiques ». Le fait qu'à leur arrivée en Égypte, les Britanniques se soient heurtés à une tradition française déjà bien établie a certainement été la raison principale de la non-application de la politique juridique « indienne».

Pourquoi la politique « indienne » a-t-elle donc réussi au Soudan? Est-ce en raison de l'absence de tradition juridique contraire au droit anglais, ou en raison de l'absence de toute tradition juridique? On sait qu'à l'époque où le Soudan était sous autorité ottomano-égyptienne, le pouvoir était très centralisé (époque turkiyya, de 1821 à 1884). Il est toutefois difficile de savoir quelle loi dominait (Köndgen, 1992, p. 15-16). Mustafa (1971, p. 37-38) a trouvé des éléments allant dans le sens d'une application de la loi 
islamique et des Codes militaire et civil égyptiens. La situation est plus claire pour l'époque mahdiyya, de 1881 à 1898, période intermédiaire entre le gouvernement ottomano-égyptien et l'autorité anglo-égyptienne, pendant laquelle le droit islamique a prédominé (Köndgen, 1992, p. 16-18). Cette primauté du droit islamique montre qu'à leur arrivée, les Britanniques se trouvèrent face à une situation similaire à celle qu'ils avaient connue en Inde.

En Égypte ce fut différent. Ils y trouvèrent un droit qui avait commencé à s'égyptianiser mais qui était encore français dans une très large mesure. Par conséquent, les officiers coloniaux britanniques qui, comme Cramer, avaient servi en Inde, ne se trouvèrent pas face à une tradition juridique locale - comme ils auraient pu s'y attendre - mais étrangère. Le fait que cette tradition était celle de leur grand rival n'est pas la moindre des raisons expliquant leur antipathie à son égard, alors même qu'ils ne la connaissaient peut-être même pas. À cette époque, le droit comparé en tant que discipline juridique n'en était encore qu'à ses débuts. Les Britanniques préféraient ce qu'ils aimaient ; et ils aimaient ce qu'ils connaissaient, que cela vienne d'Angleterre ou d'Inde.

31 De plus, ils ne parvinrent pas à trouver en Égypte le personnel qui, traditionnellement, est considéré comme nécessaire à l'application du droit islamique : le cadi et le mufti, ulémas formés en droit islamique. Comme Cromer le fait remarquer, ils avaient été remplacés dans une large mesure par des juristes formés en France ou en Égypte, au sein d'écoles et collèges nouvellement créés, totalement indépendants d'al-Azhar. Les fonctions au sein des tribunaux mixtes et nationaux étaient considérées comme exigeant un type d'expertise différent de celui traditionnellement dispensé par l'éducation islamique. Par conséquent, un nouveau dispositif juridique avait été mis en place, comprenant des institutions comme des barreaux d'avocats et des facultés de droit, afin d'organiser la profession juridique à l'intérieur et à l'extérieur des tribunaux (Botiveau, 1989, p. 235-236).

Cromer n'a donc qu'à moitié raison lorsqu'il estime que les tribunaux mixtes et nationaux étaient français et donc étrangers à l'Égypte. À l'époque où il a formulé cette appréciation, le droit du statut personnel, domaine du droit auquel les gens peuvent le plus facilement s'identifier, était encore du ressort des tribunaux islamiques. Par conséquent, il n'y avait pas de différence à ce niveau-là entre l'Égypte, l'Inde ou le Soudan (Robinson, 1987, p. 258-259). Il est possible que Cromer ait raison lorsqu'il affirme que la réception du droit français risquait d'entrâner de grands préjudices sociaux. Mais il en aurait été de même en cas de réception du droit anglo-indien. Pour Hirsch (1981, p. 11 -28), une réception juridique est un processus social. Elle ne peut se produire du jour au lendemain. Elle ne se traduit jamais par un acte unique mais toujours par un long processus, d'une durée au moins égale à. une génération.

La complexité d'un processus de réception juridique est due à la différence qui existe entre l'idéal que peut représenter une norme juridique et sa réalité. Idéalement, une norme juridique fournit ce qui est supposé être. En réalité, ce qui est supposé être ne correspond pas nécessairement à la façon dont une norme juridique est acceptée ou interprétée. Une réception juridique n'est pas un transfert de normes mais bien le transfert des idées qui sont derrière ces normes. Elle doit être perçue comme telle, sinon elle échouera et sera source de dommages. L'idée derrière une norme ne Rappliquant pas automatiquement à la réalité d'un contexte juridique nouveau et une réception impliquant en principe une traduction, un échec dû à une mauvaise 
traduction ne peut être écarté. En cas d'échec, la norme doit être rectifiée ou écartée totalement. Cela peut conduire à de grandes difficultés et à des malentendus, particulièrement au début d'un processus de réception.

Qu'il y ait déjà eu un début de réception du droit civil et commercial français en Égypte, avant même la création des tribunaux mixtes et nationaux, voilà sans doute ce qui constitue le fait le plus significatif. En 1856, les majâlis al-tujjâr s'étaient vu doter d'une division d'appel ${ }^{12}$. Un an plus tard, on leur demanda d'appliquer le Code commercial ottoman de $1850^{13}$, qui reprenait les parties I et III du Code de commerce français de 1807 et traitait du commerce en général ainsi que de l'insolvabilité et de la faillite ${ }^{14}$. S'ils ne pouvaient y trouver la solution à un problème, les majâlis devaient se référer au Code civil français de 1804. Cette disposition prenait en considération le fait que le Code de commerce français n'était pas un Code indépendant mais complémentaire, et ne pouvait fonctionner sans le Code civil ${ }^{15}$. Par conséquent, avant même sa réception par les tribunaux mixtes et nationaux, le droit civil et commercial français avait déjà été appliqué par des juridictions égyptiennes. Au moment où Cramer critiquait le droit civil français comme étranger à l'Égypte, ce droit avait commencé depuis plus d'une génération son processus d'égyptianisation.

\section{BIBLIOGRAPHIE}

ARMINJON Pierre, NOLDE Boris (Baron) et WOLFF Martin, 1950-1952, Traité de Droit Comparé, 3 vol., Paris, LGDJ.

BERGER Morroe, 1957, Bureaucracy and Society in Modem Egypt: A Study of the Higher Civil Service, Princeton, Princeton U.P.

BOTIVEAU Bernard, 1989, «L'adaptation d'un modèle français d'enseignement du droit au Proche-Orient ", dans M. Flory et J. R. Henry (dir.), L'enseignement du droit musulman, Paris, Éd. du CNRS, p. 229-252.

BRINTON Jasper Yeates, 1968, The Mixed Courts of Egypt, 2nd ed., New Haven, Yale U.P.

BROWN Nathan, 1997, 777e Rule of Law in the Arab World: Courts in Egypt and the Gulf, Cambridge, Cambridge U.P.

CARONI Pio, 1988, « Privatrecht »: Eine sozialhistorische Einführung, Baie/Francfort, Verlag Helbig \& Lichtenhahn.

CROMER (Earl of), 1908, Modern Egypt, 2 vol., Londres, Macmillan.

DAVID René, 1964, Les grands systèmes de droits contemporains, Paris, Dalloz.

FAHMY Khaled, 1997, All the Pasha's Men: Mehmed Ali, His Army and the Making of Modern Egypt, Cambridge, Cambridge U.P.

GOLDBERG Jan, 1997, « L'Europe au-delà de l'Europe : réflexions sur l'entrée de l'Égypte dans la famille française du droit », dans Gilles Boëtsch, Baudouin Dupret et Jean-Noël Ferrié (dir.), Droits 
et sociétés dans le Monde arabe: Perspectives socio-anthropologiques, Aix-en-Provence, Presses Universitaires d'Aix-Marseille.

HAMED Raouf Abbas, 1995, « The Siyasatname and the Institutionalization of Central Administration under Muhammad 'Ali ", in Nelly Hanna (éd.), The State and its Servants: Administration in Egypt from Ottoman Times to the Present, Cairo, AUC Press, p. 75-86.

HIRSCH Ernst E., 1981, Rezeption als sozialer ProzeB: Erläutert am Beispiel der Türkei, Berlin, Duncker \& Humblot.

ISMAIL Orner Sid Ahmed, 1982, Zur Entwicklung des sudanesischen Rechts vor allem auf dem Gebiet des Deliktsrechts aus übernommenem englischen Recht,thèse, Université de Hambourg, Hambourg.

KHÂNKî 'Azîz, 1939, Al-Mahâkim al-mukhtalita wa-l-mahâkim al-ahliyya, Le Caire, al-Matba'a al-'asriyya.

KÖNDGEN Olaf, 1992, Das islamisierte Strafrecht des Sudan : Von seiner Einführung 1983 bis Juli 1992, Hambourg, Deutsches Orient-Institut.

KRÜGER Hilmar, 1987, « Überblick über das Privatrecht der Staaten des Àgyptischen Rechtskreises ", Recht van de Islam, $\mathrm{n}^{\circ} 5$.

LAURENS Henry et al., 1989, L'Expédition d'Égypte, 1798-1801, Paris, Armand Colin.

MANSFIELD Peter, 1973, The British in Egypt,Newton Abbot, Victorian Book Club.

MUSTAFA Zaki Mustafa, 1971, The Common Law in the Sudan: An Account of the «Justice, Equity and Good Conscience » Provision, Oxford, Oxford U.P.

PATRA Atul Chandra, 1962, The Administration of Justice under the East-India Company in Bengal, Bihar and Orissa,Londres, Asia Publishing House.

PETERS Rudolph, 1997, « Islamic and Secular Criminal Law in Nineteenth Century Egypt: The Role and Function of the Qadi ", Islamic Law and Society, $\mathrm{n}^{\circ}$ 4, p. 70-90.

REIMER Michael John, 1997, Colonial Bridgehead: Government and Society in Alexandria, 1807-1882,Boulder/Oxford, Westview.

RHEINSTEIN Max :

- 1954, (éd.), Max Weber on Law in Economy and Society, traduit par Edward Shilset Max Rheinstein, Cambridge, MA, Harvard U.P.

- 1987, Einführung in die Rechtsvergleichung, 2e éd., Reimer von Borries (éd.), Munich, Beck.

ROBINSON Francis, 1987, « Säkularisierung im Islam », dans Wolfgang Schluchter (éd.), Max Webers Sicht des Islams : Interpretation und Kritik, Francfort, Suhrkamp, p. 256-271.

SAROUFIM Ebeid, 1949, England and the Criminal Legislation of Egypt from 1882, thèse, Oxford University, Oxford.

SCOTT John, 1899, «Judicial Reform in Egypt ", Journal of the Society of Comparative Legislation, N.S., 1(1899)2, p. 240-252.

ZWEIGERT Konrad et KÖTZ Hein, 1987, An Introduction to Comparative Law,2nd ed., trans. Tony Weir, Oxford, Oxford U. P. 


\section{NOTES}

1. Cf. Codes des Tribunaux Mixtes d'Égypte, précédés du Règlement d'organisation judiciaire, Alexandrie, L Carrière, 1896.

2. Pour le texte des Codes nationaux, cf. Bulletin des lois et décrets: année 1883, Le Caire, Jules Barbier, 1883.

3. Selon Krüger, le Code a été reçu à des degrés divers en Syrie, Irak, Libye, au Koweït, au Qatar, en Somalie, Algérie, Jordanie, Afghanistan, au Yémen, aux Émirats et à Bahreïn. Même le Soudan a essayé à deux reprises de l'adopter, en 1971 et 1984.

4. Si l'on en croit Raouf Abbas Hamed (1995), l'identité de cet expert n'est pas claire. Les sources arabes le nomment « Rosah ». Pour le texte du qânûn al-siyâsatnâmah, cf. Ahmad Fathî Zaghlûl, alMuhâmà, Le Caire, Matba'at al-ma'ârif, 1900, appendices, p. 4-26.

5. Pour le texte du qânûn al-muntakhabât, cf. Zaghlûl, op. cit., appendices, p. 100-155.

6. Zaghlûl, op. cit., p. 185-187.

7. Citation traduite de l'anglais.

8. Traduit de l'anglais. Art. 27 de la Règle 11,1772, tel que cité dans M. Barry Hooker, Legal Pluralism : An Introduction to Colonial and Neo-colonial Laws, Oxford, Oxford U.P., 1975, p. 61.

9. Ibid.

10. Traduit de l'anglais.

11. Traduit de l'anglais.

12. Cf. tartîb al-qanâsil, dans Zaghlûl, op. cit., appendices, p. 43-45.

13. Cf. lâ'ihat majlis al-appello, dans ibid., appendices, p. 45-52.

14. Pour le texte du Code commercial ottoman, cf. Législation ottomane, Grégoire Aristarchi (éd.), Constantinople, Demetrius Nicolaïdes, 1874, vol. 1, p. 275-343.

15. Sur les relations entre le droit civil et le droit commercial, cf. Caroni (1988, p. 168-176).

INDEX

Mots-clés : droit, droit français, occupation britannique, XIXe siècle, colonisation

\section{AUTEURS}

JAN GOLDBERG

St Antony's College, Oxford 